\title{
Gender and Age Effects on Ventricular Repolarization Abnormality in Japanese General Carriers of a G643S Common Single Nucleotide Polymorphism for the $K C N Q 1$ Gene
}

\author{
Tomoya Ozawa, MD; Makoto Ito, MD; Shinji Tamaki, MD; Takenori Yao, MD; \\ Takashi Ashihara, MD; Yoshikuni Kita, PhD*; Tomonori Okamura, MD*; \\ Hirotsugu Ueshima, MD*; Minoru Horie, MD
}

\begin{abstract}
Background The KCNQ1 single nucleotide polymorphism (SNP), G643S, is known to be associated with secondary long QT syndrome (LQTS) and to cause a mild reduction in KCNQ1 current. However, the precise incidence and its association with QT intervals remain unknown in the greater cohort of the population in Japan. Methods and Results The genotype was screened at codon 643 of KCNQ1 in 992 residents of a farming community. Eighty-eight individuals (female/male $=52 / 36,8.9 \%$ ) were found to have a heterozygous G643S SNP. Matching both gender and age, we randomly selected 243 control (G643G) cases and compared the electrocardiogram parameters in both groups; QT, QTf (QT corrected by Fridericia's formula) intervals, the peak and the end of the T wave (Tpe) interval, and the Tpe/QT ratio. The latter 2 reflect the transmural dispersion of ventricular repolarization (TDR). In G643S carriers, both Tpe and Tpe/QT were significantly longer than in noncarriers, without significant QT prolongation. Both genders showed a tendency for an increase in QTf with aging. In females, both Tpe and Tpe/QT showed a similar significant increase with age, which was not observed in males.

Conclusions In elderly females, G643S might be an independent risk factor for secondary LQTS by causing a greater TDR. (Circ J 2006; 70: 645-650)

Key Words: Electrocardiography; KCNQ1; Secondary LQTS; Single nucleotide polymorphism; Transmural dispersion of repolarization
\end{abstract}

$A$ potassium channel gene, $K C N Q 1$, encodes the $\mathrm{a}$-subunit of the slow delayed rectifier $\mathrm{K}$ (IKs) channel with accessory protein, MinK (coded by $K C N E 1)$ ! Since the first report on the relationship between KCNQ1 mutations and congenital long QT syndrome (LQTS), , more than 120 genetic variations have been identified in $K C N Q 13,4$ Among these genetic variations, a transposition of guanine to adenine at the 1727 nucleotide, causing G643S, was found to be a single nucleotide polymorphism (SNP) in $\sim 11 \%$ of the Japanese general population and has been shown to cause a very mild reduction in IKs reconstituted in the mammalian cell line, 5 This SNP, therefore, appeared to be a risk factor predisposing the secondary LQTS. The relationship between the G643S genotype and the phenotype in regard to electrocardiogram (ECG), however, remains unclear. In the present study, we screened the SNP among 992 habitants in a farming community (Shigaraki) near Kyoto, Japan, and examined the characteristics of the ECG parameters in both SNP-positive and negative individuals.

(Received December 19, 2005; revised manuscript received February 21, 2006; accepted March 9, 2006)

Departments of Cardiovascular and Respiratory Medicine, *Department of Health Science, Shiga University of Medical Science, Otsu, Japan

Mailing address: Tomoya Ozawa, MD, Department of Cardiovascular Medicine, Shiga University of Medical Science, Otsu 520-2192,

Japan. E-mail: ozawat@iris.eonet.ne.jp
More recently, in addition to the absolute QT interval, transmural dispersion of repolarization (TDR) has been evaluated by measuring the interval between the peak and the end of the T wave (Tpe) and the Tpe/QT ratio?-10 These TDR parameters have drawn our attention in regard to the occurrence of torsades de pointes (TdP) in LQTS!1-14 Therefore, in the present study, we measured these TDR indicators along with conventional QT intervals in genotyped G643S carriers and compared these results with those in non-carriers. The incidence of cardiac events in patients with congenital LQTS has been known to vary, dependent on both gender and age. For example, young male and elderly female LQT1 patients have a higher risk of having cardiac events 15,16 This is also true for the acquired type of LQTS; victims of TdP tend to be elderly females6,17-18 Taken together, gender and age could largely influence the phenotype induced by a genetic variant, especially in cases where the functional change resulting from the variant was subtle 19 We therefore analyzed the ECG parameters of G643S carriers and non-carriers by dividing the subjects of the study into several groups depending on their gender and age.

\section{Methods}

DNA Isolation and Genotyping

Among 2,902 individuals who entered the Shigaraki Study 20992 were enrolled to the present study; others were 


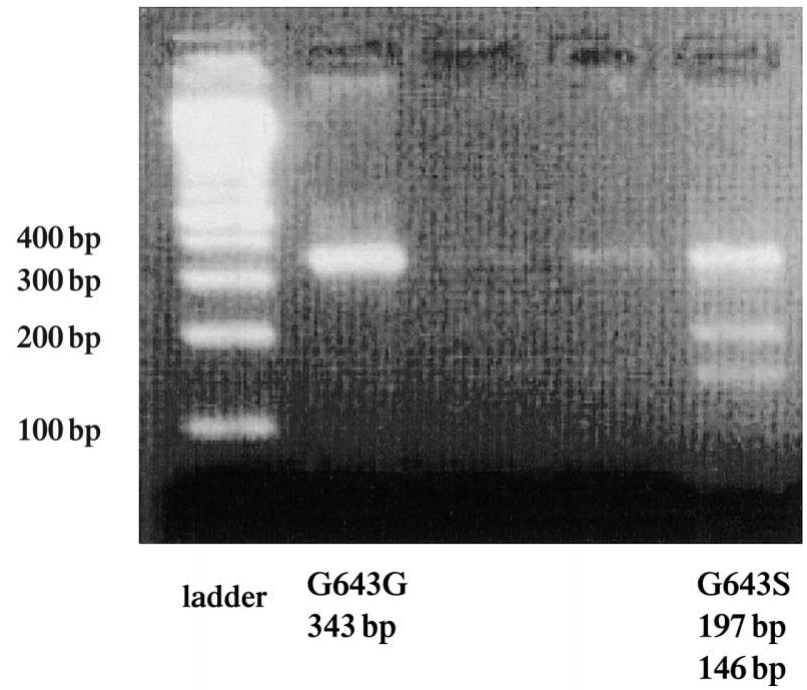

Fig 1. Electrophoresis of G643G and G643S polymerase chain reaction (PCR) products. The PCR products were electrophoresed on a $2.0 \%$ Agarose gel (Nippon Gene, Japan), and observed for a 343 base pair (bp) fragment (KCNQ1 G643G genotype), and both $146 \mathrm{bp}$ and 197 bp fragments (KCNQ1 G643S genotype).

excluded from the analysis because of current drug intake, diabetes mellitus and a history of cardiac diseases. Genomic DNA was isolated from peripheral leukocytes, and the $K C N Q 1$ genotype at codon 643 was determined by using a restriction enzyme. Briefly, for the genomic DNA from each individual (extracted by using a DNA extraction Kit; Wako, Japan), a set of primers encompassing the DNA fragment containing codon 643 was used for polymerase chain reaction (PCR) (sense: 5'-ACTCATCACCGACATGCTTCACCAGCT, anti-sense: 5'-CTTTTAGGAGGTGGCCTCCTTCAGA). The presence of a SNP was determined by finding whether the restriction endonuclease, Pst-I (Takara, Japan), cleaved the PCR product (Fig 1). Finally, the correlation between the electrophoresis pattern and the genotype was confirmed by using a direct sequencing method (ABI PRISM 310 Genetic Analyzer, Parkin-Elmer, USA).

\section{Case and Control Definition}

Among the 992 participants, 88 had the G643S SNP (36 males, 52 females) and 904 had the G643G wild genotype (344 males, 560 females). Thus, the heterozygous genotype was estimated to be $8.9 \%$ ( 88 out of 992). In the 88 SNP carriers, 7 subjects, 2 males and 5 females, showed abnormal 12-lead ECG at rest (eg, ischemic change, conduction abnormalities, atrial fibrillation, non-specific ST change, or left ventricular hypertrophy) and were excluded from the analysis. Finally, the SNP group was comprised of 81 individuals (34 males, 47 females). For each individual G643S carrier, 3 control cases were randomly drawn from G643G carriers by matching both gender and age (102 males, 141 females). None of these control cases showed abnormal ECG. The protocol used in the present study was approved by the Institutional Review Board of Shiga University of Medical Science (Nos. 11-15, 1999).

\section{ECG Measurements}

All ECG parameters were measured manually (Fig 2). QT was defined as the interval between the QRS onset and the end of the $\mathrm{T}$ wave, at the point where the isoelectric line intersected a tangential line drawn on the maximal downslope of the positive T wave. Q-Tpeak (QTp) was defined as the interval between the QRS onset and the peak of the T wave. Then, the interval between the peak and the end of the $\mathrm{T}$ wave (Tpe) was calculated as QT minus QTp, and Tpe/QT was calculated as the relative Tpe interval divided by the QT interval. The interval of the Tpe has been shown to reflect the TDR ? $^{-9}$ We examined the characteristics of the ECG parameters using the V5 lead, because it is known that the unipolar lead reflects the local electric potential gradient of the free wall at the left ventricle?,8 Measurements were performed as the mean of approximately 3 consecutive beats by 2 investigators who were unaware of the subject's status. There were no significant differences in the measured numerical data obtained by the 2 investigators.

\section{Statistical Analysis}

Data are presented as mean \pm SD. Multivariate regression analysis was used for the comparison of each ECG parameter over 4 effects (genotype, gender, heart rate (HR), and age; Table 1). The non-paired 1-tail Student's t-test was used to compare the unpaired parameters (HR, age, and ECG parameters) between the different groups (Tables 2-4;

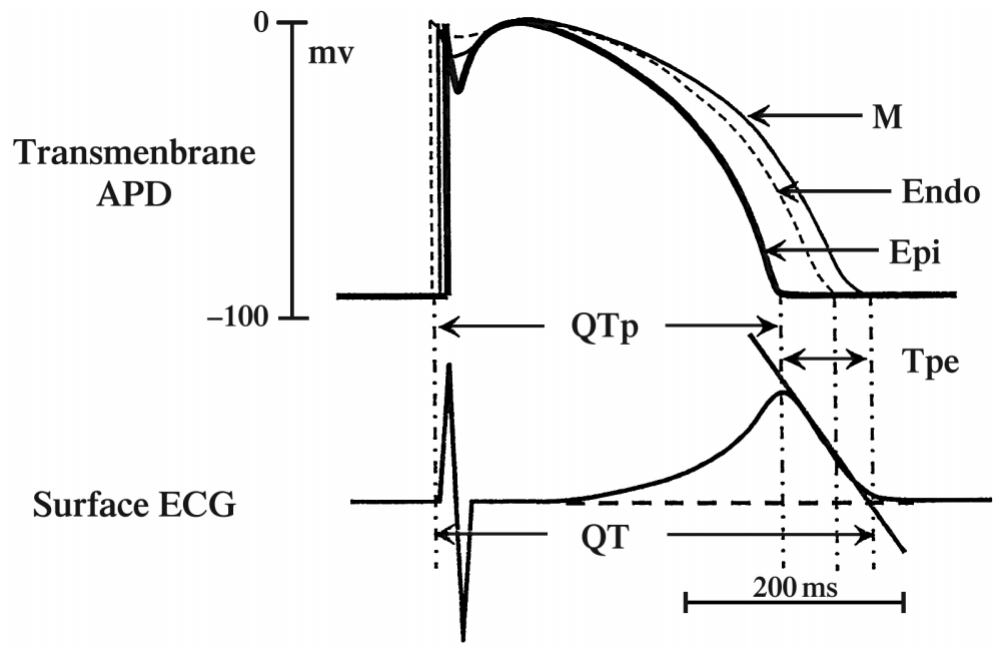

Fig 2. Variety of action potential duration (APD) in each myocardium layers and the electrocardiogram (ECG) repolarization parameters. Endo, the APD of the endocardial layer; M, the APD of the subendocardial layer; Epi, the APD of the epicardial layer; QTp, the interval between the Q-point and the T-wave peak; Tpe, QT interval-Q-Tpeak interval. 
Table 1 Multivariate Regression Analysis of Repolarization Parameters for Each Category

\begin{tabular}{|c|c|c|c|c|c|c|c|c|c|c|}
\hline \multirow{2}{*}{ Category } & \multicolumn{2}{|c|}{$Q T$} & \multicolumn{2}{|c|}{$Q T c$} & \multicolumn{2}{|c|}{ QTf } & \multicolumn{2}{|c|}{ Tpe } & \multicolumn{2}{|c|}{$T p e / Q T$} \\
\hline & F ratio & $p$ value & F ratio & $p$ value & F ratio & $p$ value & F ratio & $p$ value & F ratio & $p$ value \\
\hline Genotype & 0.010 & $N S$ & 0.263 & $N S$ & 0.181 & $N S$ & 12.760 & $<0.001$ & 12.465 & $<0.001$ \\
\hline Gender & 4.779 & $<0.05$ & 19.276 & $<0.001$ & 5.106 & $<0.05$ & 1.217 & $N S$ & 3.777 & 0.052 \\
\hline$H R$ & 293.8 & $<0.0001$ & 86.146 & $<0.001$ & 0.218 & $N S$ & 0.044 & $N S$ & 28.209 & $<0.0001$ \\
\hline Age & 5.842 & $<0.05$ & 9.197 & $<0.01$ & 11.610 & $<0.001$ & 6.870 & $<0.01$ & 2.527 & $N S$ \\
\hline
\end{tabular}

QTc, $Q T / R R^{1 / 2}$; QTf, $Q T / R R^{1 / 3} ;$ Tpe, QT interval-Q-T peak interval; HR, heart rate.

The data were derived from 136 males and 188 females, Shigaraki town, Shiga, Japan, 1999.

Table 2 Characteristic Status in Each Genotype

\begin{tabular}{lcccccccc}
\hline \hline Genotype & $\begin{array}{c}\text { Age } \\
\text { (years) }\end{array}$ & $\begin{array}{c}H R \\
\text { (beats/min })\end{array}$ & $\begin{array}{c}Q T \\
(\mathrm{~ms})\end{array}$ & $\begin{array}{c}Q T c \\
(\mathrm{~ms})\end{array}$ & $\begin{array}{c}Q T f \\
(\mathrm{~ms})\end{array}$ & $\begin{array}{c}Q T p \\
(\mathrm{~ms})\end{array}$ & $\begin{array}{c}\text { Tpe } \\
(\mathrm{ms})\end{array}$ & Tpe/QT \\
\hline G643S $(n=81)$ & $54.0 \pm 14.5$ & $64.3 \pm 9.4$ & $394.1 \pm 26.7$ & $405.3 \pm 22.3$ & $401.3 \pm 19.4$ & $306.1 \pm 28.1$ & $88.0 \pm 12.3$ & $0.224 \pm 0.034$ \\
G643G $(n=243)$ & $54.4 \pm 15.5$ & $63.7 \pm 8.9$ & $394.3 \pm 26.8$ & $404.1 \pm 22.8$ & $400.6 \pm 20.2$ & $312.3 \pm 27.4$ & $82.0 \pm 13.3$ & $0.209 \pm 0.034$ \\
p value & $N S$ & $N S$ & $N S$ & $N S$ & $N S$ & $N S$ & $p<0.0001$ & $p<0.0001$ \\
\hline
\end{tabular}

Data are mean $\pm S D$. Probability values between G643S carriers and non-carriers.

QTp, Q-T peak interval; G643S, carriers; G643G, non-carriers. Other abbreviations see in Table 1.

The data were derived from 136 males and 188 females, Shigaraki town, Shiga, Japan, 1999.

Table 3 Characteristic Status in Each Gender-Genotype

\begin{tabular}{|c|c|c|c|c|c|c|c|}
\hline & $\begin{array}{c}\text { Age } \\
\text { (years) }\end{array}$ & $\begin{array}{c}H R \\
\text { (beats/min) }\end{array}$ & $\begin{array}{l}Q T \\
(m s)\end{array}$ & $\begin{array}{l}Q T c \\
\text { (ms) }\end{array}$ & $\begin{array}{l}\text { QTf } \\
\text { (ms) }\end{array}$ & $\begin{array}{l}\text { Tpe } \\
\text { (ms) }\end{array}$ & $\begin{array}{c}T p e / Q T \\
\text { (ms) }\end{array}$ \\
\hline \multicolumn{8}{|l|}{ G643S carriers } \\
\hline Male $(n=34)$ & $53.2 \pm 16.6$ & $60.1 \pm 9.2$ & $397.1 \pm 28.3$ & $394.6 \pm 17.9$ & $395.2 \pm 17.1$ & $89.2 \pm 9.3$ & $0.226 \pm 0.029$ \\
\hline Female $(n=47)$ & $54.5 \pm 13.1$ & $67.1 \pm 8.5$ & $392.0 \pm 25.7$ & $412.7 \pm 22.1$ & $405.5 \pm 20.0$ & $87.2 \pm 14.0$ & $0.223 \pm 0.037$ \\
\hline$P_{1}$ & NS & $p<0.001$ & $N S$ & $p<0.001$ & $p<0.05$ & NS & $N S$ \\
\hline \multicolumn{8}{|l|}{ G643G non-carriers } \\
\hline Male $(n=102)$ & $53.3 \pm 16.1$ & $61.2 \pm 9.4$ & $397.3 \pm 24.8$ & $398.7 \pm 21.4$ & $398.0 \pm 17.3$ & $81.1 \pm 12.0$ & $0.204 \pm 0.031$ \\
\hline Female $(n=141)$ & $54.9 \pm 15.3$ & $65.7 \pm 7.9$ & $391.7 \pm 27.8$ & $408.0 \pm 22.7$ & $402.3 \pm 21.7$ & $83.1 \pm 14.1$ & $0.212 \pm 0.035$ \\
\hline$P_{2}$ & NS & $p<0.001$ & NS & $p<0.05$ & NS & NS & $p<0.05$ \\
\hline$P_{3} / P_{4}$ & $N S / N S$ & NS/NS & $N S / N S$ & $N S / N S$ & $N S / N S$ & $p<0.001 / N S$ & $p<0.001 / N S$ \\
\hline
\end{tabular}

Data are mean $\pm S D . P_{1}$ and $P_{2}$ are the probability values, male vs female, in each genotype, respectively; $P_{3} / P_{4}, G 643 S$ vs $G 643 G$ in male/in female, respectively.

Abbreviations see in Table 1.

The data were derived from 136 males and 188 females, Shigaraki town, Shiga, Japan, 1999.

Table 4 Demography and Characteristic Status of Each Gender

\begin{tabular}{lcccccc}
\hline \hline & \multicolumn{7}{c}{ Age subset (years) } \\
\cline { 2 - 7 } & $<31$ & $31-40$ & $41-50$ & $51-50$ & $61-70$ & $>70$ \\
\hline Number & & & & & & \\
$\quad$ Male & 16 & 24 & 24 & 16 & 40 & 16 \\
$\quad \begin{array}{l}\text { Female } \\
\text { Mean age (years) }\end{array}$ & 20 & 16 & 36 & 36 & 52 & 28 \\
$\quad$ Male & $25.0 \pm 4.0$ & $36.8 \pm 2.8$ & $47.0 \pm 2.6$ & $56.2 \pm 2.6$ & $65.8 \pm 2.5$ & $73.7 \pm 3.9$ \\
$\quad$ Female & $27.9 \pm 3.0^{\S}$ & $35.1 \pm 2.2^{*}$ & $46.5 \pm 3.3^{*}$ & $56.9 \pm 2.6^{*}$ & $65.1 \pm 2.6^{*}$ & $74.4 \pm 3.3^{*}$ \\
HR (beats/min) & $62.3 \pm 12.2$ & $60.1 \pm 8.5$ & $61.3 \pm 9.3$ & $63.6 \pm 8.9$ & $59.1 \pm 8.6$ & $62.2 \pm 10 / 0$ \\
$\quad$ Male & $66.4 \pm 6.6^{*}$ & $66.9 \pm 10.3^{\S}$ & $67.0 \pm 7.7^{\S}$ & $65.0 \pm 8.6^{*}$ & $65.5 \pm 8.1^{\S}$ & $66.7 \pm 7.9^{*}$ \\
$\quad$ Female & & & & & &
\end{tabular}

Data are mean $\pm S D .{ }^{s} p<0.05 ; * p=N S$ vs female in each age subset.

Figs 3-5). Univariate regression analysis was used for comparisons of the repolarization parameters and age (Fig 5). A probability value of $<0.05$ was considered significant.

\section{Results}

Characteristics of ECG Parameters: Genotype Effects on TDR

For the 992 total cases, the resting HR ranged from 41 to
91 beats/min., and the age from 19.1 to 85.4 years. There was no significant correlation between HR and age. There was no subject having a QTc $>480 \mathrm{~ms}^{21,22} \mathrm{~T}$-waves of G643S carriers showed a broad-based pattern, and there was no case with obvious bifid $\mathrm{T}$-waves among all carriers and control cases.

Table 1 summarizes the correlation between the ECG parameters and genotype (G643S or G643G), gender, HR or age. QT, QTc (Bazett's correction formula $=\mathrm{QT} / \mathrm{RR}^{1 / 2}$ ), 
A
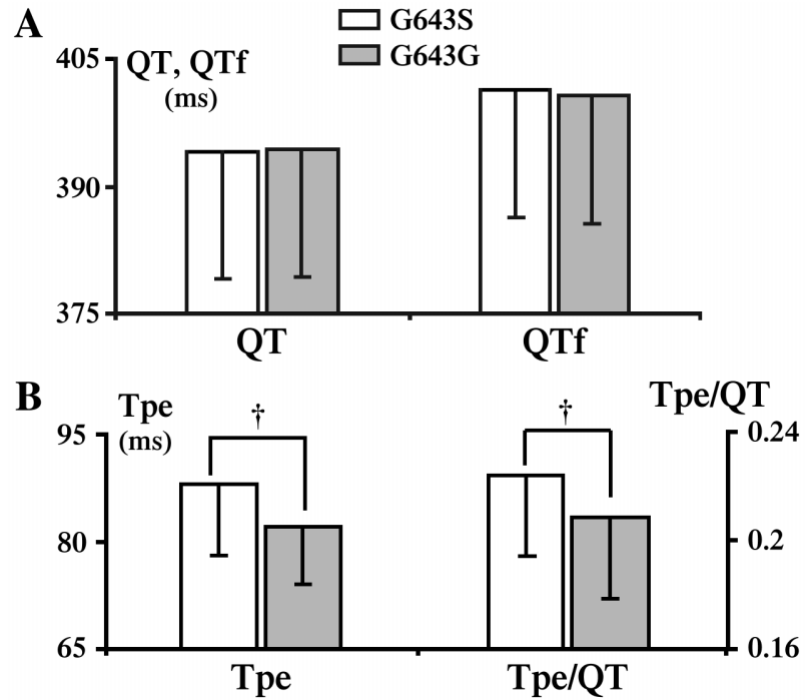

Fig 3. Two genotypes and electrocardiogram repolarization parameters. The relationship between the intervals of each ventricular repolarization; QT, QTf (A), Tpe, and Tpe/QT (B), and the genotype are presented for G643S carriers (blank portion) and G643G non-carriers (shaded portion). QTf indicated QT/RR ${ }^{1 / 3}$; Tpe, QT interval-QTpeak interval (Q-Tpeak, the interval between the Q-point and the T-wave peak); Tpe/QT, the relative value of Tpe divided by QT; G643S, carriers; G643G, non-carriers. Data are presented as mean \pm SD. ${ }^{\mathrm{p}}<0.0001$.

and Tpe/QT showed a strong correlation with HR, but Tpe remained independent of HR? ${ }^{23}$ Therefore, absolute Tpe values were adopted for examination, without correction by $\mathrm{HR}$, and Tpe/QT was used as the relative fractional proportion to the QT interval. The present study population showed a wide range of HR, and it is known that QTc is strongly affected by HR, and that HR is strongly affected by gender ${ }^{24,25}$ Indeed, in the present study cohort, HR affected QTc but not Fridericia's correction formula $(\mathrm{QTf}=$ QT/RR ${ }^{1 / 3}$ ), whereas gender affected both QT corrections
(Table 1). It could be said that QTf had a net relationship with gender, irrespective of HR. We therefore used QTf in the following analyses.

The genotype at codon 643 of $K C N Q 1$ showed a significant correlation with 2 repolarization parameters, Tpe and Tpe/QT (Table 1). Fig 3 and Table 2 summarize the comparison of the ECG parameters between the 2 genotypes. Tpe and Tpe/QT were significantly larger in G643S than in G643G carriers, although there were no significant differences of QT or QTf intervals between the 2 genotypes.

\section{Different Genotype Effects on Repolarization Parameters in Each Gender}

The QT and QTf values showed a significant correlation with gender and age in all of the study population (Table 1). We divided the 2 genotype groups by gender, resulting in 4 subsets. Table 3 and Fig 4 summarize the characteristics of each repolarization parameter in these 4 subsets. In both genotype groups, the mean QT interval was longer in males than in females. This was because HR was significantly lower in males (Table 3), and there was HR-dependent prolongation of the QT interval26 In contrast, the mean QTf interval was longer in females (Table 3), and the female G643S subset showed a significantly longer QTf interval than the male G643S subset ( $p=0.02)$. There was no difference in Tpe and Tpe/QT between the 2 genders. However, between the 2 genotypes, Tpe and Tpe/QT values were longer in the G643S group than in the control. This was significant only between the 2 male groups $(\mathrm{p}<0.02)$ (Figs 4B,C).

\section{TDR Increases With Age, Especially in Female Groups}

In our initial analyses of the correlation between categorical and ECG parameters in 324 individuals (Table 1), it was found that age was significantly correlated with the ECG parameters. Fig 5 shows a summary of the age-dependent changes in 4 ECG parameters in the 2 gender groups, and the demography and characteristics of each gender are presented in Table4. QTf, Tpe and Tpe/QT showed a tendency to increase prominently with age in females. Similar

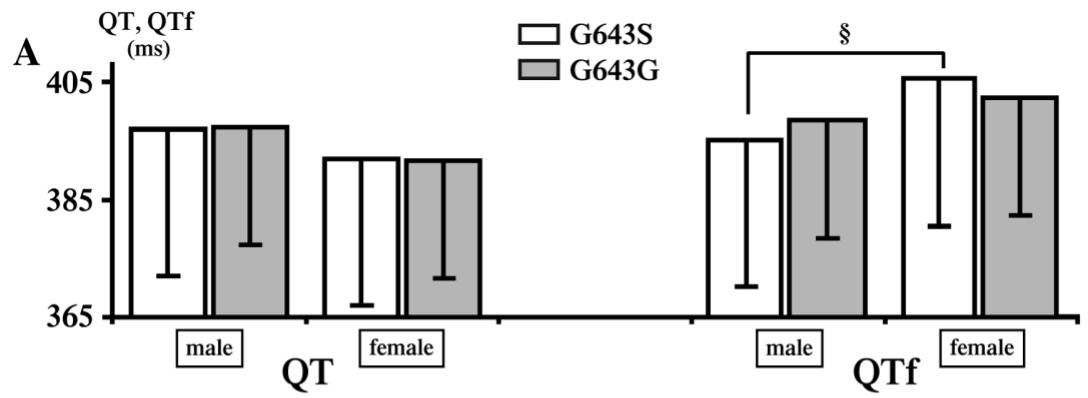

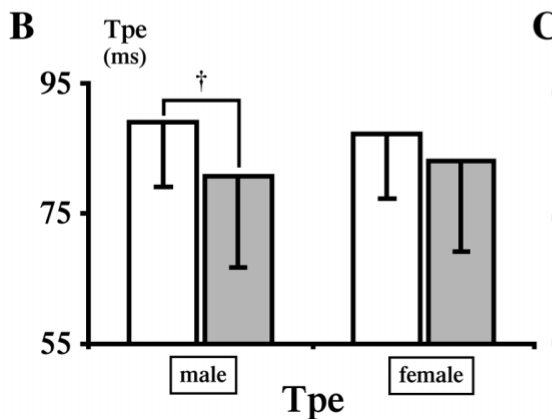

C Tpe/QT

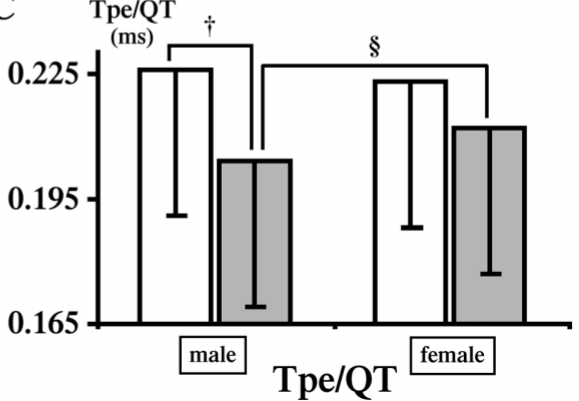

Fig 4. Gender-dependent analyses on genotype-electocardiogram (ECG) parameter relationship. The relationship between the intervals of each ventricular repolarization; QT, QTf (A), Tpe (B), and Tpe/QT (C), and the genotype in each gender are presented for G643S carriers (blank portion) and G643G non-carriers (shaded portion). QTf indicated $\mathrm{QT} / \mathrm{RR}^{1 / 3}$; Tpe, QT interval-QTpeak interval (Q-Tpeak, interval between the Q-point and the T-wave peak); Tpe/QT, Tpe divided by QT; G643S, carriers; G643G, non-carriers. Data are presented as mean \pm SD. ${ }^{\S} \mathrm{p}<0.05 ;{ }^{\dagger} \mathrm{p}<0.0001$. 
A

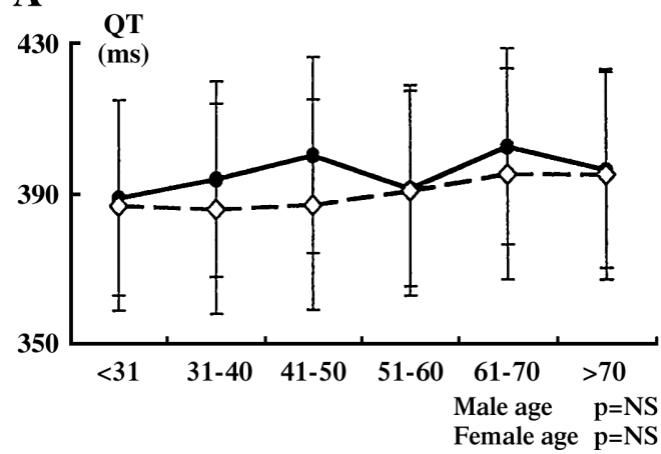

C Tpe

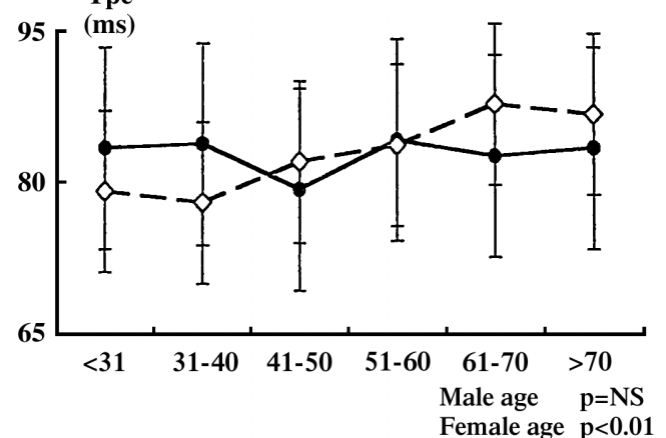

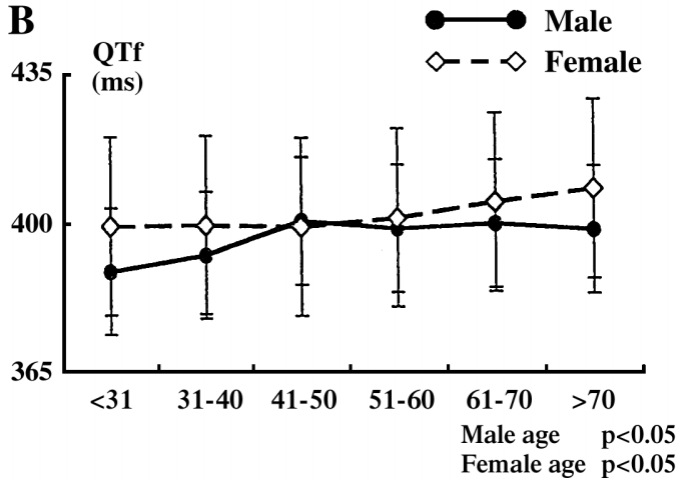

D Tpe/QT

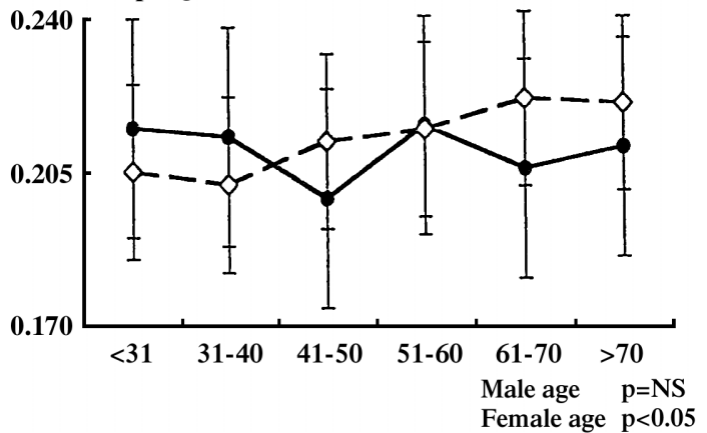

Fig 5. Age-dependent analyses on the electrocardiogram (ECG) parameters. The correlations of the intervals of each ventricular repolarization; QT (A), QTf (B), Tpe (C), and Tpe/QT (D), and ages are presented for the male carriers (solid line and $\bullet$ ) and for the male non-carriers (dotted line and $\diamond)$ of different ages. QTf indicated QT/RR ${ }^{1 / 3}$; Tpe, QT interval-Q-Tpeak interval (Q-Tpeak, the interval between the Q-point and the T-wave peak); Tpe/QT, Tpe divided by QT. Data are presented as mean $\pm \mathrm{SD}$; $\mathrm{p}$ showed the probability value of univariate regression analysis between age and each ECG repolarization parameter in male or female groups, respectively.

age-dependent increases in QTc and Tpe were previously reported in healthy Japanese volunteers! ${ }^{10}$ Because G643S SNP was associated with greater TDR-related ECG parameters (Figs 3,4), the arrhythmogenic effects of G643S appeared to be even stronger in the elderly female subgroup.

\section{Discussion}

In the present study we demonstrated that the $K C N Q 1$ gene SNP, G643S, has a relatively high incidence rate $(8.9 \%)$ in a Japanese community population (Shigaraki) and that the heterozygous SNP carriers showed a significantly greater Tpe and Tpe/QT ratio compared to the control group selected by matching both gender and age (Fig 3). In addition, in elderly females, the TDR-related parameters including QTf were greater than in the other 3 groups, although they did not reach statistical significance (Fig 4). Thus, a larger TDR would be a risk factor for ventricular arrhythmias in SNP-positive elderly females.

In comparison with the degree of QT prolongation, Tpe and Tpe/QT values were greater in the SNP group, suggesting the presence of an abbreviation of the QTp interval. In the functional assay using a heterologous expression system, G643S polymorphism was found to be functional and caused a mild reduction in IKs-like currents that were co-expressed with MinK encoded by KCNE1 (by 30\% in the heterozygous condition). As shown in the scheme of Fig 2, the Tpe interval was obtained as the difference between the QT and QTp intervals. Meanwhile, previous studies $^{8,11}$ suggested that the net QT interval reflects the action potential duration (APD) in the subendocardial (M) layer, and the QTp interval reflects the APD in the epicardial layer. Therefore, the Tpe interval corresponds to the differences in the APDs between the 2 layers (or TDR) ${ }^{7-9}$

In human myocardium, electrophysiological studies have demonstrated that both epicardial and endocardial cells have stronger net inward repolarizing currents (as a result of strong IKs) compared to $\mathrm{M}$ cells, which is caused primarily to relatively weak IKs in the M layer. ${ }^{27}$ In contrast, a rapidly activating component of the delayed rectifier (IKr) is distributed homogeneously in all layers and more predominantly than IKs.28 It has also been suggested that the transient outward current, Ito, is more abundant in the epicardial layer than in the 2 other layers ${ }^{29}$ Therefore, outward potassium conductance is most scarce in the M layer, and a very mild reduction in IKs may cause a greater APD prolongation in this layer, while the epicardial APD might remain constant or even be abbreviated because other $\mathrm{K}$ current systems such as IKs and Ito could compensate for it. Roden proposed a similar mechanism as a "repolarization reserve" that modifies the arrhythmogenesis ${ }^{30}$ This might partially serve to explain the reason why the extension of the Tpe interval was seen without any significant QT prolongation appearing in ECGs obtained at rest.

Supposing that compensating outward K currents were decreased by the presence of additional risks such as hypokalemia and drugs associated with QT prolongation, 19 the arrhythmia risk for the SNP carriers could increase markedly. Indeed, we reported that 6 out of 95 LQTS patients 
(6.3\%) had the heterozygous G643S SNP but no mutations in $K C N Q 1, K C N H 2, S C N 5 A$, and $K C N E 1$ ? Interestingly, the probands were all female with the acquired form of LQTS (mean age of 42 years). In some of these subjects, TdP was triggered by bradycardia and hypokalemia. Other unidentified genetic and/or epigenetic factors could collaborate in unmasking the latent vulnerability to arrhythmias (prolongation of QT interval or augmentation of TDR), thereby predisposing the SNP carriers to TdP.

\section{Study Limitations}

The number of SNP carriers was relatively low. When we analyzed the age-dependent effect on the ECG parameters (Fig 5), the correlation was therefore examined in the whole population. Cases under 18 years old were not included in the present study, and therefore the characteristics and the G643S SNP effect on the ventricular repolarization in childhood and adolescence were not determined. However, because the SNP was associated with the secondary type of LQTS, this was not an essential problem.

In conclusion, in our cohort of 324 individuals, $K C N Q 1$ G643S SNP appeared to be associated with the greater progression of the TDR-related parameters, especially in the elderly female group. It is in our hope that other prospective studies will be conducted to clarify the secondary LQTS mechanisms and gender differences.

\section{Acknowledgments}

The study was supported by Research Grants from the Ministry of Education, Culture, Sports, Science, and Technology (12204059, 06670414, $02454211,16209025,3812112,17790485)$ and by Health Science Research Grants from the Japan Ministry of Health, Labor, and Welfare, and the Japan Society for the Promotion of Science (Grant-in-aid for Scientific Research: 16590500, 2812113, 2812127).

\section{References}

1. Sanguinetti MC, Curran ME, Zou A, Shen J, Spector PS, Atkinson DL, et al. Coassembly of $K V L Q T 1$ and MinK (IsK) proteins to form cardiac IKs potassium channel. Nature 1996; 384: 80-83.

2. Wang Q, Curran ME, Splawski I, Burn TC, Millholland JM, VanRaay TJ, et al. Positional cloning of a novel potassium channel gene: KvLQT1 mutations cause cardiac arrhythmias. Nat Genet 1996; 12: $17-23$.

3. Splawski I, Shen J, Timothy KW, Lehmann MH, Priori S, Robinson JL, et al. Spectrum of mutations in long-QT syndrome genes: KvLQT1, HERG, SCN5A, KCNE1, and KCNE2. Circulation 2000; 102: $1178-1185$.

4. Priori SG, Napolitano C. Gene Connection for the Heart [homepage on the internet]. Pavia, Italy: European Society of Cardiology; c2000-01. Available from: http://pc4.fsm.it:81/cardmoc/

5. Iwasa $H$, Itoh $T$, Nagai $R$, Nakamura $Y$, Tanaka $T$. Twenty single nucleotide polymorphisms (SNPs) and their allelic frequencies in four genes that are responsible for familial long QT syndrome in the Japanese population. J Hum Genet 2000; 45: 182-183.

6. Kubota T, Horie M, Takano M, Yoshida H, Takenaka K, Watanabe $\mathrm{E}$, et al. Evidence for a single nucleotide polymorphism in the $K C N Q 1$ potassium channel that underlies susceptibility to life-threatening arrhythmias. J Cardiovasc Electrophysiol 2001; 12: $1223-$ 1229.

7. Shimizu W, Antzerevitch C. Sodium channel block with mexiletine is effective in reducing dispersion of repolarization and preventing Torsade de pointes in LQT2 and LQT3 models of the long-QT syndrome. Circulation 1997; 96: 2038-2047.

8. Yan GX, Antzerevitch C. Cellular basis for the normal T wave and the electrocardiographic manifestations of the long-QT syndrome. Circulation 1998; 98: 1928-1936.
9. Aiba T, Shimizu W, Inagaki M, Hidaka I, Tatewaki T, Sunagawa K. Transmural heterogeneity of the action potential configuration in the feline left ventricle. Circ J 2003; 67: 449-454.

10. Nakagawa M, Takahashi N, Watanabe $M$, Ichinose $M$, Nobe $S$, Yonemochi $\mathrm{H}$, et al. Gender differences in ventricular repolarization: Terminal $\mathrm{T}$ wave interval was shorter in women than in men. Pacing Clin Electrophysiol 2003; 26: 59-64.

11. Roden DM, Lazzara R, Rosen M, Schwartz PJ, Towbin J, Vincent GM. Multiple mechanism in the long QT syndrome: Current knowledge, gaps, and future directions: The SADS Foundation Task Force on LQTS. Circulation 1996; 94: 1996-2012.

12. Shimizu W, Antzerevitch C. Cellular basis for the ECG features of the LQT1 from the long-QT syndrome: Effects of beta-adrenergic agonists and antagonists and sodium channel blockers on transmural dispersion of repolarization and Torsade de Pointes. Circulation 1998; 98: 2314-2322.

13. Di Diego JM, Belardinelli L, Antzerevitch C. Cisapride-induced transmural dispersion of repolarization and Torsade de Pointes in the canine left ventricular wedge preparation during epicardial stimulation. Circulation 2003; 108: $1027-1033$.

14. Takenaka K, Ai T, Shimizu W, Kobori A, Ninomiya T, Otani H, et al. Exercise stress test amplifies genotype-phenotype correlation in the LQT1 and LQT2 forms of the Long-QT syndrome. Circulation 2003; 107: 838-844.

15. Moss AJ, Schwartz PJ, Crampton RS, Tzivoni D, Locati EH, MacCluer J, et al. The long QT syndrome: Prospective longitudinal study of 328 families. Circulation 1991; 84: 1136-1144.

16. Schwartz PJ, Priori SG, Spazzolini C, Moss AJ, Vincent GM, Napolitano C, et al. Genotype-phenotype correlation in the long-QT syndrome: Gene-specific triggers for life-threatening arrhythmias. Circulation 2001; 103: 89-95.

17. Ebert SN, Liu XK, Woosley RL. Female gender as a risk factor for drug-induced cardiac arrhythmia: Evaluation of clinical and experimental evidence. J Womens Health 1998; 7: 547-557.

18. Makkar RR, Fromm BS, Steinmman RT, Meissner MD, Lehmann $\mathrm{MH}$. Female gender as a risk factor for Torsade de Pointes associated with cardiovascular drugs. JAMA 1993; 270: 2590-2597.

19. Members of the Sicilian Gambit. The Search for Novel Antiarrhythmic Strategies. Jpn Circ J 1998; 62: 633-648.

20. Tamaki S, Nakamura Y, Tsujita Y, Nozaki A, Amamoto K, Kadowaki $\mathrm{T}$, et al. Polymorphism of the angiotensin converting enzyme gene and blood pressure in a Japanese general population (the Shigaraki Study). Hypertens Res 2002; 25: 843-848.

21. Schwartz PJ, Moss AJ, Vincent GM, Crampton RS. Diagnostic criteria for the long QT syndrome: An update. Circulation 1993; 88: $782-784$.

22. Priori SG, Schwartz PJ, Napolitano C, Bloise R, Ronchetti E, Grillo $\mathrm{M}$, et al. Risk stratification in the long-QT syndrome. $N$ Engl J Med 2003; 348: $1866-1874$.

23. O'Donnell J, Knoebel SB, Lovelace DE, McHenry PL. Computer quantitation of Q-T and terminal $\mathrm{T}$ wave (aT-eT) intervals during exercise: Methodology and results in normal men. Am J Cardiol 1981; 47: 1168-1172.

24. Bazett HC. An analysis of time relations electrocardiograms. Heart 1920; 7: 353-370.

25. Funck-Brentano C, Jaillon P. Rate-correlated QT interval: Techniques and limitations. Am J Cardiol 1993; 72: 17B-22B.

26. Rautaharju PM, Zhou SH, Wong S, Calhoun HP, Berenson GS, Prineas R, et al. Sex differences in the evolution of the electrocardiographic QT interval with age. Can J Cardiol 1992; 8: 690-695.

27. Liu DW, Antzerevitch C. Characteristics of the delayed rectifier current (Ikr and IKs) in canine ventricular epicardial, mid myocardial and endocardial myocytes: A weaker Iks contributes to the longer action potential of the M cell. Circ Res 1995; 76: 351-365.

28. Veldkamp MW. Is the slowly activating component of delayed rectifier current: Iks, absent from undiseased human ventricular myocardium? Cardiovasc Res 1998; 40: 433-435.

29. Drici MD, Burklow TR, Haridasse V, Glazer RI, Woosley RL. Sex hormones prolong the QT interval and downregulate potassium channel expression in the rabbit heart. Circulation 1996; 94: 1471 1474.

30. Roden DM. Taking the "idio" out of "idiosyncratic": Predicting torsades de pointes. Pacing Clin Electrophysiol 1998; 21: 10291034. 